\title{
De la spécificité du concept de santé environnementale vis-à-vis du développement durable
}

On the specificity of the concept of environmental health vis-à-vis sustainable development

\section{Julien Forbat}

\section{OpenEdition}

Journals

Édition électronique

URL : http://journals.openedition.org/developpementdurable/10241

DOI : 10.4000/developpementdurable.10241

ISSN : 1772-9971

Éditeur

Association DD\&T

\section{Référence électronique}

Julien Forbat, « De la spécificité du concept de santé environnementale vis-à-vis du développement durable », Développement durable et territoires [En ligne], Vol. 5, n² I Juin 2014, mis en ligne le 20 juin 2014, consulté le 19 avril 2019. URL : http://journals.openedition.org/developpementdurable/10241 DOI : 10.4000/developpementdurable.10241

Ce document a été généré automatiquement le 19 avril 2019

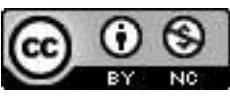

Développement Durable et Territoires est mis à disposition selon les termes de la licence Creative Commons Attribution - Pas d'Utilisation Commerciale 4.0 International. 


\title{
De la spécificité du concept de santé environnementale vis-à-vis du développement durable
}

\author{
On the specificity of the concept of environmental health vis-à-vis sustainable \\ development
}

Julien Forbat

1 Dans un contexte où le développement durable a acquis une influence considérable dans le débat entourant la conduite des politiques publiques (Meadowcroft, 2007 ; Jordan, 2008), quelle place accorder à la santé environnementale, un concept justement tiraillé entre, d'une part, sa proximité avec celui de développement durable et, d'autre part, une singularité promouvant un rapport particulier de l'homme à son environnement? S'agitil d'un concept véritablement distinct, d'un sous-concept exprimant certaines dimensions du développement durable ou d'un concept largement redondant? Le but de notre article est de répondre à ces questions.

Dans un premier temps, nous nous intéresserons au concept de santé, dans la mesure où ce dernier développe une distinction entre "disease" et "illness" ${ }^{1}$ qui structure de manière déterminante les diverses formulations du concept de santé environnementale.

Ensuite, nous aborderons les acceptations actuelles de la santé environnementale dont nous verrons qu'elles suivent, schématiquement, deux logiques. La première se caractérise par une approche relativement biomédicale et sectorielle et tend à se situer dans un rapport de subordination avec le concept de développement durable. La seconde s'appuie davantage sur la notion de bien-être dans une optique largement systémique et intersectorielle.

4 Finalement, après avoir distingué ces deux types de conceptualisation, nous tenterons de clarifier l'intérêt de la santé environnementale en tant que concept vis-à-vis du développement durable, en identifiant certaines sources fécondes de reformulation de celle-ci, de sorte à en offrir une définition pertinente. 
5 Alors que l'histoire des concepts de santé environnementale et de développement durable remonte à l'Antiquitée , plusieurs éléments contribuent, à partir des années 1960, d'un côté, à la résurgence des préoccupations de santé environnementale et, de l'autre, à la consécration du concept de développement durable. En effet, les menaces apparaissent comme pesant non plus seulement sur les milieux naturels mais sur la survie de l'Homme elle-même. Le développement du nucléaire, la médiatisation de plusieurs catastrophes écologiques majeures (naufrage du Torrey Canyon en 1967 par exemple), la publication de livres dénonçant les atteintes causées par la société de consommation à la nature et à la santé humaine (Carson, 1962) et l'influence de mouvements sociaux (contre-culture américaine dans un premier temps), favorisent l'essor des mouvements écologistes qui verront, dès le début des années 1970, se développer parallèlement l'écologie politique. De manière générale, l'importance accrue accordée aux enjeux du développement durable et de la santé environnementale est fortement liée, dans les pays occidentaux, au passage d'une société basée sur des principes relatifs au bien-être matériel et à la sécurité physique à une société visant à promouvoir davantage la qualité de vie (Inglehart, 1977).

6 En conséquence, nous étudierons la période allant de la seconde moitié du XX ${ }^{\text {ème }}$ siècle à nos jours. Pour ce faire, nous puiserons abondamment dans la littérature émanant des institutions internationales traitant du développement durable et de la santé environnementale avant de faire appel à certains auteurs offrant une vision critique des concepts en question. En effet, souvent considéré durant les décennies récentes comme une composante du développement durable (à l'instar du premier principe de la Déclaration de Rio en 1992), le concept de santé environnementale procède toutefois d'un développement historique qui lui est propre. Les difficultés de plusieurs institutions internationales à intégrer les enjeux de santé environnementale à leurs programmes sont d'ailleurs en partie liées à une prise en compte réductrice de celle-ci. Ainsi, une approche critique de la construction du concept de santé environnementale nous permettra, d'une part, de mettre en exergue ses spécificités vis-à-vis du développement durable, soit une forme d'interdisciplinarité et un cadre institutionnel particuliers ainsi qu'une approche davantage anthropocentrique. D'autre part, cette autonomisation du concept de santé environnementale nous permettra de décrire les conditions d'une intégration renforcée de ses composantes, tant en termes de types d'environnement que de déterminants de la santé pris en compte.

\section{Santé et développement durable : une intégration artificielle?}

7 Cette partie est consacrée à la genèse des concepts de santé environnementale et de développement durable. Elle vise notamment à mettre en exergue leur difficile articulation durant la seconde moitié du XX ${ }^{\text {ème }}$ siècle. La première section traite donc des évolutions qu'a connues le concept de santé, tandis que la seconde insiste sur les limites du rapprochement conceptuel entre santé environnementale et développement durable.

\subsection{La santé selon l'OMS : un concept à géométrie variable}

8 Dès 1946, soit deux ans avant la création formelle de l'Organisation mondiale de la Santé (OMS), la Conférence internationale de la Santé adopte un texte d'une importance fondamentale au regard de la définition qu'il donne de la santé : 
9 «La santé est un état de complet bien-être physique, mental et social, et ne consiste pas seulement en une absence de maladie ou d'infirmité. » (OMS, 1946)

10 Cette définition ouvre la voie à une prise en compte élargie des facteurs influençant la santé, proposant une vision systémique englobant les facteurs non-biomédicaux. En fait, cette importance accordée à la notion de bien-être traduit le passage d'une approche biomédicale reposant sur le diagnostic par les médecins et autres spécialistes de la santé de maladies ("disease") auxquelles correspondent des traitements spécifiques (la maladie est interprétée selon une logique objective/mesurable), à une approche faisant de la maladie ("illness") un concept largement dépendant de la perception des individus quant à leur situation personnelle en termes de problèmes de santé (logique subjective/vécue), la maladie devenant dès lors un concept potentiellement très vaste (Wikman, Marklund and Alexanderson, 2005).

11 Au cours de la seconde moitié du XX ${ }^{\text {ème }}$ siècle, l'OMS a développé un concept de santé systémique, reposant sur une interdisciplinarité forte et visant une large intersectorialité des politiques de santé publique. Ainsi, la volonté d'élargir le sens donné aux notions de santé et de maladie est confirmée en 1978 lors de la conférence d'Alma-Ata. À cette occasion, le caractère nécessairement intersectoriel des politiques de santé est mis en avant, ces dernières devant inclure tous les acteurs susceptibles d'influencer l'état de santé d'une population et non uniquement les spécialistes du domaine médical. La déclaration qui est adoptée va même au-delà en insistant sur le droit des individus à participer à l'élaboration et à la mise en œuvre des politiques de santé (OMS, 1978), une évocation du caractère transdisciplinaire de la santé dans la mesure où celle-ci vise notamment l'intégration contextuelle des savoirs locaux (Klein, 2004).

Cependant, la mise en œuvre de ce concept novateur - dans la mesure où il dépasse les visions traditionnellement biomédicales de la santé, héritées notamment de l'hygiénisme apparu au XIX ${ }^{\text {ème }}$ siècle ${ }^{3}$ - s'est heurtée à certaines résistances, notamment de la part des États occidentaux durant les années 1980 et 1990 qui verront dans la participation citoyenne aux programmes de santé un moyen de faire reposer sur les individus une partie des coûts de la santé jusque-là supportés par l'État (Vos et al., 2009). Par la suite, cette tension entre conceptions biomédicale et systémique influencera la formulation du concept de santé environnementale et ses liens avec celui de développement durable.

\subsection{La santé environnementale : une dimension du développement durable?}

13 Cependant, les travaux de l'OMS concernent avant tout l'accès aux soins primaires, c'està-dire qu'une part considérable des problématiques de santé existantes, notamment dans les pays développés, n'est qu'effleurée par ceux-ci. Dans ces conditions, l'émergence du concept de santé environnementale n'apparaît guère que comme la tentative de compléter après coup le contenu du développement durable, ce dernier semblant offrir un cadre conceptuel à même d'englober l'ensemble des défis, qu'ils soient sanitaires ou non, auxquels les pays développés et en voie de développement font face. D'ailleurs, la première mention du terme "développement durable", par l'Union internationale pour la conservation de la nature dans sa stratégie consacrée à la protection des ressources biologiques de la planète, se fait en dehors de toute référence à la santé (IUCN, 1980). 
14 D'un point de vue chronologique, les premiers jalons d'une définition internationale de la santé environnementale précèdent leurs équivalents relatifs au concept de développement durable, puisqu'il faut attendre 1972 et la Conférence de Stockholm pour les voir une première fois mentionnés. La déclaration qui y est adoptée par les NationsUnies sur le thème de l'environnement humain décrit ce dernier selon des perspectives relevant tant de la santé environnementale - importance des environnements naturel et construit $^{4}$ - que du développement durable - selon les principes qui tendront à le caractériser par la suite, quand bien même ces derniers recouvrent des interprétations divergentes (Zaccaï, 2002), notamment les principes de solidarité générationnelle et intergénérationnelle ou le principe d'un mode de gouvernance incluant l'ensemble des acteurs concernés (ONU, 1972). Toutefois, les formulations ultérieures des organisations internationales marquent un fléchissement dont les points d'orgue seront le Rapport Brundtland et la Conférence de Rio de 1992.

15 En 1987, les Nations-Unies utilisent pour la première fois le terme de « développement durable » dans un rapport officiel, le Rapport Brundtland. Ce dernier reste célèbre pour la définition qu'il donne du développement durable :

16 "Le développement durable est un mode de développement qui répond aux besoins du présent sans compromettre la capacité des générations futures de répondre aux leurs. Deux concepts sont inhérents à cette notion :

17 - le concept de "besoins", et plus particulièrement des besoins essentiels des plus démunis, à qui il convient d'accorder la plus grande priorité

18 - l'idée des limitations que l'état de nos techniques et de notre organisation sociale impose sur la capacité de l'environnement à répondre aux besoins actuels et à venir. " (Commission mondiale sur l'environnement et le développement, 1987)

19 Cette définition reprend des éléments apparus précédemment - en 1972 à Stockholm ou dans la stratégie de l'UICN par exemple - et leur confère une actualité nouvelle ainsi qu'une légitimité accrue, se traduisant par une popularité jamais démentie depuis, cette définition s'étant imposée dans le monde entier (Mebratu, 1998: 502). En outre, elle met explicitement en lien les enjeux de développement au sein des pays en voie de développement (PVD) avec les impacts du fonctionnement des sociétés industrialisées sur leur environnement (naturel), laissant présager du grand écart conceptuel à venir autour de la santé environnementale. 
La Suisse a adopté en 1998 un Plan d'Action Environnement Santé (PAES) qu'elle a mis en œuvre entre 2001 et 2007. Ce plan, principalement conçu par les offices fédéraux de la santé publique et de l'environnement, s'inspire presque exclusivement des définitions internationales de la santé environnementale, à l'instar de celles issues de la Conférence de Rio (1992) ou de la 2ème Conférence européenne sur l'environnement et la santé (1994). Surtout, le PAES est présenté comme "un plan d'action sectoriel, se [référant] au chapitre 6 de l'Agenda 21 [...et] comme [un] complément à la stratégie prévue pour un développement durable» (OFSP and OFEFP, 1997). Cette approche illustre évidemment l'idée selon laquelle la santé environnementale serait un sous concept du développement durable et s'explique notamment par le fait que les travaux des institutions internationales représentent, pour une administration nationale en situation de faible capacité d'expertise telle que la Section santé \& environnement de l'office fédéral de la santé publique, une source importante de légitimation et de mise en conformité (Finnemore and Sikkink, 1998).

En 1992, l'ONU organise la Conférence des Nations-Unies sur l'environnement et le développement à Rio de Janeiro. De cette réunion sont issus plusieurs documents fondamentaux relativement aux concepts de développement durable et de santé environnementale. Parmi ceux-ci, la Déclaration de Rio sur l'environnement et le développement réitère l'importance d'un certain nombre de principes élaborés au cours des années 1970 et 1980. Ainsi, le premier principe de la Déclaration cite implicitement la santé environnementale :

"Les êtres humains sont au centre des préoccupations relatives au développement durable. Ils ont droit à une vie saine et productive en harmonie avec la nature." (ONU, 1992b)

À nouveau, le développement durable y est cité comme allant de pair avec, entre autres, les principes de solidarité générationnelle et intergénérationnelle, de participation citoyenne ou encore de précaution, la santé environnementale étant quant à elle fortement associée à l'environnement naturel.

Pour sa part, le programme Action 21 («Agenda 21 » en anglais) détaille une série d'objectifs et d'actions à mener dans chacune des thématiques identifiées comme se rapportant aux enjeux de développement durable. Le chapitre 6 décrit ainsi les aspects à prendre en compte dans le cadre de la protection et de la promotion de la santé (cf. encadré 1), selon une approche se concentrant largement sur les facteurs naturels ayant une incidence en termes médicaux dans les PVD (ONU, 1992a). La santé environnementale est ici avant tout considérée comme une branche de la médecine dédiée aux maladies ("diseases") causées par l'environnement naturel (cf. encadré 2). Cette médecine environnementale ne représente pas tant un concept novateur doté de principes et pratiques originaux que la relabellisation des activités traditionnelles de soins primaires des institutions internationales, au premier rang desquelles se situe l'OMS (cf. encadré 3). 
En France, le premier Plan national santé environnement, adopté en 2004 par le gouvernement, s'inscrit assez clairement dans une logique de santé publique focalisée sur les pathologies provoquées par des facteurs de l'environnement naturel. En effet, la santé environnementale tend à y être décrite comme une "hygiène de l'environnement ", la plupart des enjeux prioritaires du plan étant à cet égard révélateurs : prévention des décès liés à la pollution atmosphérique urbaine, des cancers et des risques neurotoxiques liés aux expositions environnementales, des maladies allergiques respiratoires, protection des ressources en eau, etc. (Ministère de la Santé et de la Protection sociale et al., 2004).

Concernant le suivi des problématiques ainsi identifiées, il est intéressant de noter que l'ONU a rédigé un recueil 130 indicateurs couvrant les divers chapitres d'Action 21 (UN Commission on Sustainable Development, 1996). Or, il apparaît que, parmi les indicateurs retenus pour mesurer la santé, soit douze au total, seuls deux se rapportent à la question des formes de pollution environnementale ${ }^{5}$, en l'occurrence, ceux consacrés à la qualité de l'eau potable et à la présence de substances chimiques dans l'alimentation. Cette constatation illustre la faible opérationnalisation des enjeux de santé environnementale, compte tenu de la description qui en est faite dans les documents cadres des NationsUnies, elle-même loin d'être exhaustive.

En 1997, l'OMS entérine une nouvelle approche de la santé environnementale, considérée désormais comme une « composante essentielle du développement durable » (OMS, 1997 : 197). Cette approche, insiste sur le lien entre les enjeux du développement durable (gestion des ressources naturelles, production de déchets, etc.) et leurs effets sur la santé humaine, particulièrement dans les pays pauvres, ainsi que sur le rôle essentiel du secteur de la santé. Elle s'appuie en outre sur le modèle DPSEEA ("Driving force - Pressure - State - Exposure - Effect - Action") (Briggs, Corvalán and Nurminen, 1996) ${ }^{6}$. À la suite de ce rapport, l'OMS développe un set d'indicateurs de la santé environnementale relativement exhaustif, comparativement aux travaux réalisés jusqu'alors. Malgré les 47 indicateurs ainsi répartis en 12 thèmes, les auteurs du rapport reconnaissent certaines lacunes inhérentes à ce type d'exercice :

"There is no single set of environmental health issues, therefore; rather each issue is an artefact of the person who defines it. Nor do issues really exist in isolation; instead they connect, overlap and intersect." (OMS, 1999: 5)

Cette difficulté à traiter le caractère intersectoriel de la santé environnementale est encore plus frappante lorsqu'il s'agit d'intégrer celle-ci dans les sets d'indicateurs du développement durable. En effet, dans les documents techniques de la Commission du développement durable des Nations Unies, la santé devient une sous-catégorie de la dimension sociale du développement durable (Division for Sustainable Development, 2001), réduisant d'autant son caractère multidimensionnel et intersectoriel. 
L'OMS (2009) propose une mesure des problèmes de santé environnementale affectant la population d'un pays, la " charge de morbidité environnementale » ("environmental burden of disease"). Cette charge est calculée en Années de Vie Corrigées du facteur d'Invalidité (AVCIs), soit la combinaison des années de vie perdues en raison d'un décès prématuré et des années vécues en mauvaise santé ou avec une invalidité. Le nombre d'AVCIs imputable aux maladies environnementales s'échelonne alors entre 13 AVCIs/1 000 hab. en Islande et 289 AVCIs/1 000 hab. au Niger. Cette mesure relativement approximative ${ }^{7} \mathrm{~s}^{\prime}$ inscrit largement dans une approche médicale de lutte contre les maladies causées par des facteurs issus de l'environnement naturel.

Ainsi que les documents mentionnés le démontrent, c'est donc à partir de la fin des années 1980 que la santé environnementale commence à être davantage considérée comme une composante du développement durable, à l'instar du chapitre 6 d'Action 21, que comme un concept «indépendant». Cependant, cette intégration ne va pas sans poser plusieurs problèmes. En effet, la complexité du concept de santé environnementale ${ }^{8}$ étant largement irréductible à celle de développement durable, les tentatives de rapprochement entre ces deux concepts tendent à faire du premier une collection d'objectifs plus ou moins articulés les uns aux autres et basés sur les principes du second, une coquille vide en somme. D'ailleurs, historiquement, le premier texte employant le terme de «développement durable» (IUCN, 1980) le fait en dehors de considérations directes pour la santé humaine, soulignant le caractère indépendant des deux concepts.

\section{La santé environnementale : un nouveau paradigme}

L'un des précurseurs les plus éminents des questions de santé environnementales fut René Dubos qui, dès la fin des années 1950, proposa un cadre de réflexion novateur sur les interactions entre l'homme et son environnement, insistant notamment sur la difficulté à prendre en compte les pathologies d'origine environnementale :

"Yet it is certain that many environmental factors exert a deleterious influence on important aspects of human life. As we shall now see, the reason this danger is largely overlooked is that the damage caused to human life by environmental insults is usually so delayed and indirect that it escapes recognition through the usual analysis of cause-effect relationship." (Dubos, 1966 : 24)

31 Par la suite, et de façon presque concomitante avec les travaux de l'OMS et de l'ONU, se développe donc, principalement au sein du Bureau régional européen de l'OMS, une approche sensiblement différente de la santé environnementale, davantage complexe et axée sur les pays développés (européens). Ces préoccupations liées à la santé environnementale sont progressivement intégrées, d'abord en 1984 avec certains des objectifs inclus dans la stratégie "Santé pour tous " (OMS Bureau régional de l'Europe, 1991), puis en 1986 avec la Charte d'Ottawa mentionnant explicitement l'importance de l'environnement et appelant des ses vœux une approche « socio-écologique » de la santé (OMS, 1986). Le premier texte consacré uniquement aux interactions entre santé humaine et environnement ${ }^{10}$ - la Charte européenne de l'environnement et de la santé (OMS Bureau régional de l'Europe, 1989) - est adopté en 1989, lors de la $1^{\text {ère }}$ Conférence ministérielle sur l'environnement et la santé. Pour autant, celle-ci établit un parallèle très clair avec le développement durable. D’ailleurs, son préambule rappelle que : 
32 "[Les ministres de l'environnement et de la santé des États membres de la Région européenne de l'OMS sont] conscients de ce que le maintien et l'amélioration de la santé et du bien-être exigent un développement systématique et durable."

Ces textes débouchent en 1990 sur une définition référence de la santé environnementale :

"Environmental health comprises those aspects of human health and disease that are determined by factors in the environment. It also refers to the theory and practice of assessing and controlling factors in the environment that can potentially affect health. Environmental health, as used by the WHO Regional Office for Europe, includes both the direct pathological effects of chemical, radiation and some biological agents, and the effects (often indirect) on health and wellbeing of the broad physical, psychological, social and aesthetic environment, which includes housing, urban development, land use and transport." (OMS Bureau régional de l'Europe, 1990 : 18)

Encadré 4. Définition et usage : exemple au sein de la communauté scientifique

La définition mentionnée ci-dessus a servi de référence au projet de recherche intitulé SPHERE ("Strengthening Public Health Research in Europe") et à son volet visant à établir un état des lieux de la recherche européenne en santé environnementale (Tarkowski, 2007). Les résultats obtenus ont notamment permis de mettre en évidence quels étaient les principaux sujets couverts par la recherche entre 1995 et 2005, à savoir les liens entre santé et environnement professionnel $(36,9 \%$ des articles publiés), la question des expositions aux facteurs environnementaux $(20,8 \%)$ ou encore les maladies ("illnesses") environnementale (15\%). Cette étude a également permis de faire un certain nombre de recommandations insistant sur la nécessité pour «les politiques de santé publique d'être davantage basées sur les concepts de multi-causalité et de complexité ».

Ici sont explicitement mentionnés les effets des facteurs environnementaux compris dans un sens large, systémique, à la fois en termes de maladies diagnostiquées ("disease") et de mal-être ressenti ("illness") (cf. encadré 4), synonyme de fait d'un concept non plus subordonné au développement durable selon une interprétation biomédicale ignorant les déterminants sociaux de la santé (OMS Bureau régional de l'Europe, 1998) mais possédant une autonomie propre et reposant sur une interdisciplinarité et une intersectorialité fortes. Ce rapprochement entre "disease" et "illness" rend d'ailleurs illusoire la distinction des notions de santé et de bien-être, tant elles semblent consubstantielles, soit parce que la santé inclut le bien-être (définition de l'OMS), soit parce que le bien-être implique la santé, à l'instar de la représentation pyramidale de la santé proposée par Laughlin et Black (1995) dans laquelle il apparaît que le bien-être repose sur la réalisation de toutes les composantes intermédiaires de la santé (schéma 1). 


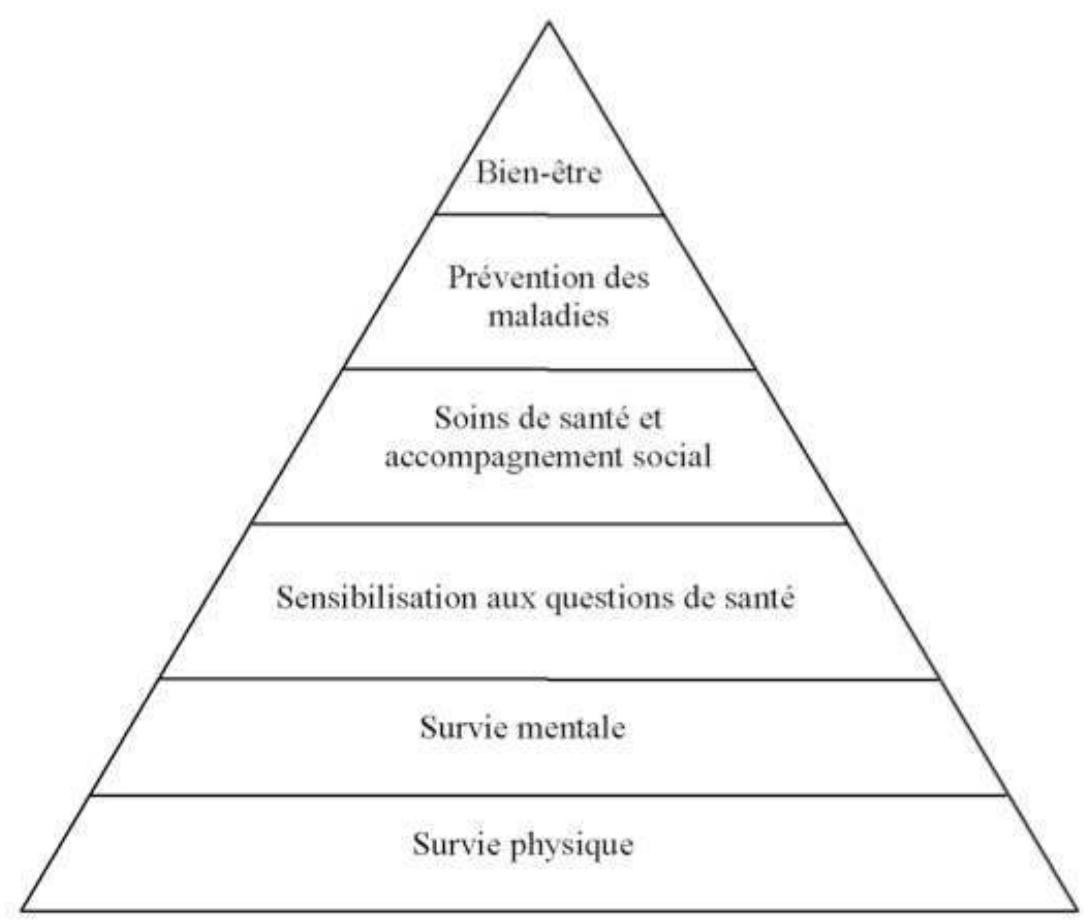

Cette distinction fondamentale entre les deux appropriations du concept de santé environnementale est d'autant plus visible dans certaines de ses représentations schématiques. En effet, celles-ci permettent de traduire avec clarté le contenu des définitions sur lesquelles elles se basent. Ainsi, en s'appuyant sur les schémas les plus représentatifs des différentes interprétations de la santé environnementale, la mise en exergue de leurs diverses composantes devient plus aisée, parce que celles-ci se trouvent rapprochées de façon synthétique, alors qu'elles peuvent être décrites de façon diffuse dans le corps d'un texte.

SCHÉMA 2 : OMS, 1946" ; COMMISSION MONDIALE SUR L'ENVIRONNEMENT ET LE DÉVELOPPEMENT, 1987 ; OMS HANCOCK, 1996
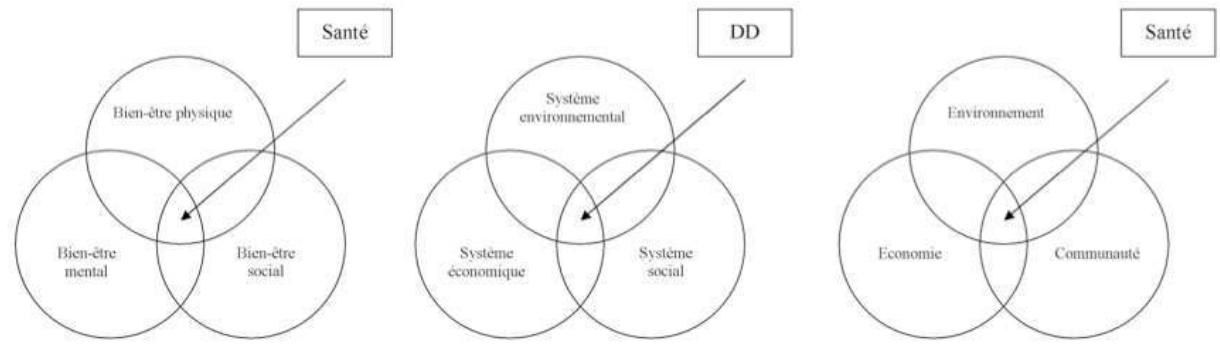

En l'occurrence, il est intéressant de remarquer les similitudes apparues au fil du temps entre représentations de la santé (OMS, 1946), du développement durable (Commission mondiale sur l'environnement et le développement, 1987) et de la santé environnementale (Hancock, 1996), traduisant certaines influences réciproques (schéma 2), puisque Hancock - s'inspirant de la représentation tridimensionnelle du développement durable - place la santé à l'intersection des dimensions économique, 
environnementale et sociale ("community"). Les trois schémas possèdent ainsi une structure commune rendant la présence de trois types de facteurs/dimensions nécessaire à la réalisation d'un concept central.

\section{La santé environnementale : un concept en devenir}

38 L'histoire des concepts de santé environnementale et de développement durable nous permet de faire un certain nombre de constatations relatives, dans un premier temps, aux liens entre les deux concepts, puis, dans un second temps, à la santé environnementale en tant que telle.

\subsection{Autonomisation du concept de santé environnementale}

Le concept de santé environnementale est à bien des égards intimement lié à celui de développement durable. Cependant, le travail de clarification du sens donné à ces deux concepts implique de procéder à une autonomisation de la santé environnementale, en mettant en exergue certaines caractéristiques lui étant propres, à savoir une forme particulière d'interdisciplinarité, un cadre institutionnel davantage spécialisé et une approche anthropocentrique marquée.

\subsubsection{Interdisciplinarité}

40 La question de l'interdisciplinarité est essentielle en ce qu'elle permet de dépasser, d'un côté, une interprétation biomédicale courante de la santé environnementale tendant à n'en faire qu'une « médecine environnementale » - c'est-à-dire une science se contentant d'étudier les atteintes provoquées par les agents physiques, chimiques et biologiques sur la santé humaine, en s'appuyant notamment sur des instruments d'évaluation du risque et d'épidémiologie (Ledrans, 2006) - et, de l'autre, une approche du développement durable principalement axée sur la protection de la nature.

41 Cependant, chacun de ces deux concepts reflète en fait une forme particulière d'interdisciplinarité. Ainsi, il existe une différence fondamentale entre la santé environnementale qui induit une « interdisciplinarité instrumentale » (Repko, $2008: 17$ ), c'est-à-dire se concentrant notamment « sur les emprunts méthodologiques et la résolution de problèmes pratiques" et le développement durable qui s'appuie sur une «interdisciplinarité conceptuelle», insistant sur "l'intégration du savoir et un questionnement en-dehors d'une base disciplinaire ». En fait, le développement durable opère comme un concept synthétique, chapeautant l'articulation des disciplines concernées entre elles. Cette distinction permet selon nous d'expliquer les diverses tentatives, bien qu'infructueuses, d'intégration du concept de santé environnementale dans celui de développement durable. Elle permet en outre de mieux saisir la dichotomie existant entre NEHAP et stratégies nationales de développement durable, les premiers procédant selon une approche davantage pragmatique que les secondes.

\subsubsection{Cadre institutionnel}

La complexité du concept de santé environnementale se traduit par un décalage important entre sa formulation au sein des institutions "spécialisées » (OMS Europe par exemple) et celles «non spécialisées » (ONU notamment). En effet, alors que les premières 
tendent à en faire un concept profondément interdisciplinaire et intersectoriel, les secondes se contentent d'une approche davantage disciplinaire, ce qui s'explique en grande partie par la volonté d'y replacer la santé environnementale dans le contexte du développement durable (intégration à la dimension sociale) et par la nécessité d'élaborer un concept valable tant pour les pays riches que pour ceux en voie de développement, d'où une tension entre la santé en tant que "besoin de sécurité » et la santé environnementale en tant que « besoin d'accomplissement personnel» (Maslow, 1943).

\subsubsection{Anthropocentrisme}

Cette volonté, somme toute récente, d'inscrire la santé environnementale dans le cadre du développement durable nous conduit à interroger la nature véritable de ces concepts. Compte tenu de leurs similitudes acquises au fil du temps - la recherche d'une approche interdisciplinaire, intersectorielle, systémique, etc. - se pourrait-il que les deux concepts ne se distinguent véritablement qu'en fonction de l'importance qu'ils accordent à l'être humain, la santé environnementale s'y consacrant exclusivement tandis que le développement durable la relativise d'après la place qu'il occupe en relation avec l'écosystème terrestre et ses composantes? En fait, la santé environnementale pourrait être qualifiée de variante la plus anthropocentrique du développement durable, dont l'homme serait la principale (seule) finalité, en dépit des velléités de certains chercheurs essayant par exemple d'intégrer santé humaine et santé animale au sein du concept de " one health" (Zinsstag et al., 2011).

Encadré 5. Définition et usage : exemple au sein d'une association citoyenne

Le Réseau Environnement Santé (RES) regroupe des associations actives dans les domaines de la santé et de la protection de la nature, des scientifiques ainsi que des professionnels de santé. Son objectif est d'œuvrer à la reconnaissance par les politiques publiques des enjeux de santé environnementale. Pour ce faire, le RES adopte une définition étendue de l'environnement qui considère « que l'environnement au sens global du terme est en cause. Alimentation, mode de vie et pollutions s'influencent réciproquement. Il faut donc considérer ces différents environnements dans leur ensemble et dans leur interrelation ", ce qui implique que "la crise sanitaire impacte l'ensemble de l'économie et du champ social» (Bousson, 2012). Nous retrouvons ici les caractéristiques du concept de santé environnementale décrit selon une approche systémique (prise en compte de plusieurs niveaux ou types d'environnement) et intégrée (prise en compte non seulement des conséquences "environnementales " mais également sociales et économiques des problèmes de santé environnementale).

\subsection{Intégration du concept de santé environnementale}

Une fois la distinction entre santé environnementale et développement durable davantage établie, il convient d'intégrer conceptuellement certaines caractéristiques de la santé environnementale jusqu'à présent cloisonnées.

\subsubsection{Environnement}

Si la santé environnementale vise à véritablement prendre en compte l'idée de bien-être, elle doit intégrer, dans une optique systémique, le plus grand nombre possible de 
déterminants de la santé (cf. encadré 5). Ce qui implique évidemment de dépasser certaines tentatives infructueuses ayant fortement circonscrit le nombre des facteurs ou des types d'environnement influant sur la santé. D'ailleurs, cette visée systémique possède indéniablement une qualité heuristique en ce qu'elle laisse ouverte la porte ouverte à l'étude de nouvelles formes d'interactions entre facteurs/environnements influençant la santé humaine. En outre, plusieurs auteurs reconnaissent que le choix des facteurs considérés comme environnementaux est en partie basé sur des critères non médicaux, à l'instar de Smith et al. qui expliquent pourquoi les facteurs liés aux styles de vie ne sont pas qualifiés de facteurs environnementaux :

"In particular, diet, including alcohol, and smoking are extremely significant risk factors for a range of important diseases and thus for total health status in many parts of the world. Yet, their inclusion as environmental risk factors would tend to overwhelm the other, more conventionally understood environmental factors." (Smith, Corvalan and Kjellström, 1999 : 573)

Schéma 3. Smith et al., 1999

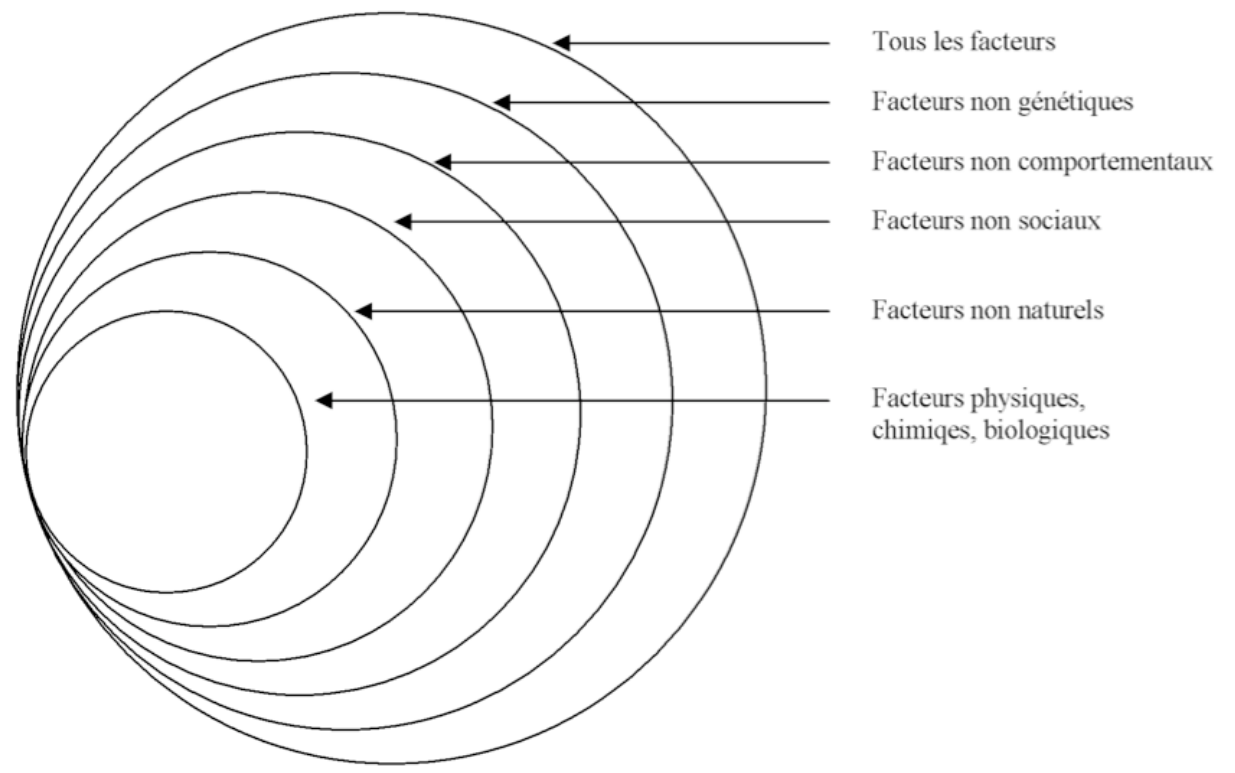

La part d'arbitraire qui préside au choix des facteurs environnementaux le conduit à distinguer les définitions plus ou moins inclusives de ces derniers, couvrant un large spectre allant de celles se concentrant sur l'environnement naturel à celles incluant tous les facteurs, y compris génétiques (schéma 3). La distinction souvent faite entre facteurs environnementaux et non environnementaux trouve également son origine dans la prégnance, au sein des cultures occidentales, de conceptions anthropocentriques - issues par exemple du christianisme ou de la Renaissance - faisant de l'Homme un être extérieur à la nature, parfois voué à la dominer (Bourdeau, 2004). Ainsi nous considérons la santé de l'être humain comme un état résultant de l'interrelation de l'ensemble de ses environnements, notamment biologique, chimique, physique ou socio-économique (Lawrence, $2004: 490$ ). 


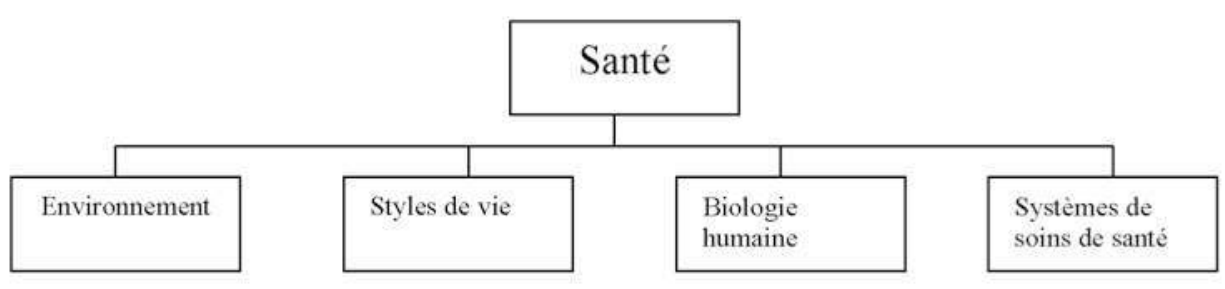

\subsubsection{Déterminants de la santé}

La distinction traditionnelle des types de facteurs influençant la santé (Lalonde, 1974) soit les facteurs environnementaux, les styles de vie, les facteurs biologiques humains (génétiques) et les systèmes de soin de santé (schéma 4$)^{12}$ - tend à occulter les interactions qui existent entre eux en adoptant une lecture sectorielle et additive (multidisciplinaire) plutôt qu'interdisciplinaire. En effet, l'épigénétique, qui étudie l'influence de l'environnement sur l'expression des gènes, a par exemple démontré un lien de cause à effet entre l'alimentation de futures mères durant des épisodes de famine et l'apparition de "marqueurs épigénétiques» chez leurs enfants, désactivant l'expression de certains gènes et provoquant plusieurs types de problèmes de santé, par exemple des propensions à l'obésité ou à la schizophrénie supérieures à la normale (Ahmed, 2010). De la même manière, il est évident que les comportements des individus sont influencés par leur environnement, la présence d'un parc à proximité de son lieu d'habitation pouvant par exemple encourager une personne à pratiquer une activité physique régulière (Perdue, Stone and Gostin, 2003). Finalement, l'influence des systèmes de soins de santé, et notamment leur accessibilité, ne peut être étudiée sans une mise en relation avec leur environnement socioéconomique et politique (Doorslaer, Masseria and Koolman, 2006). 


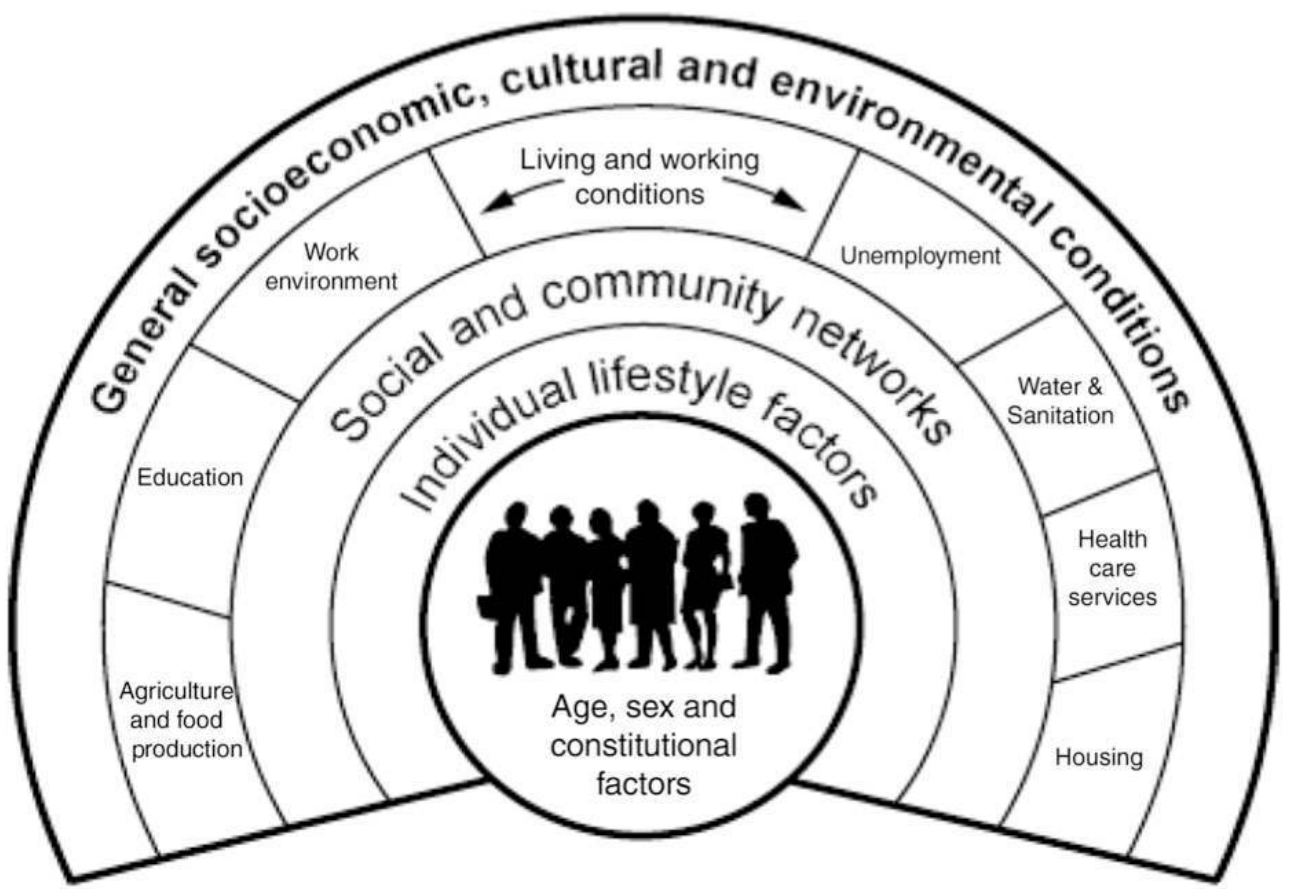

Certains auteurs proposent une complexification de la représentation des déterminants de la santé. Dahlgren et Whitehead (1991) distinguent ainsi cinq classes de déterminants imbriqués les unes dans les autres selon une logique spatiale, allant du centre (micro / individu) aux extrémités (macro / environnement général) (schéma 5). Cette conception qui sera affinée par la suite (Barton, Mitcham and Tsourou, $2003: 150$ ) vise notamment à décrire l'influence des déterminants d'ordre supérieur sur ceux d'ordre inférieur, par exemple, l'impact de l'environnement construit sur la propension des individus à pratiquer une activité physique. Or, il nous semble que les liens ainsi établis tendent à négliger une approche véritablement systémique, puisque d'éventuels effets de rétroaction sont par exemple écartés. À cet égard, mentionnons la représentation schématique proposée par Lawrence (Lawrence, 2004: 490). Celle-ci nous semble davantage refléter la nature systémique des enjeux soulevés par la santé environnementale puisqu'elle évite de hiérarchiser - si ce n'est en importance, du moins spatialement - les déterminants de la santé (schéma 6). 


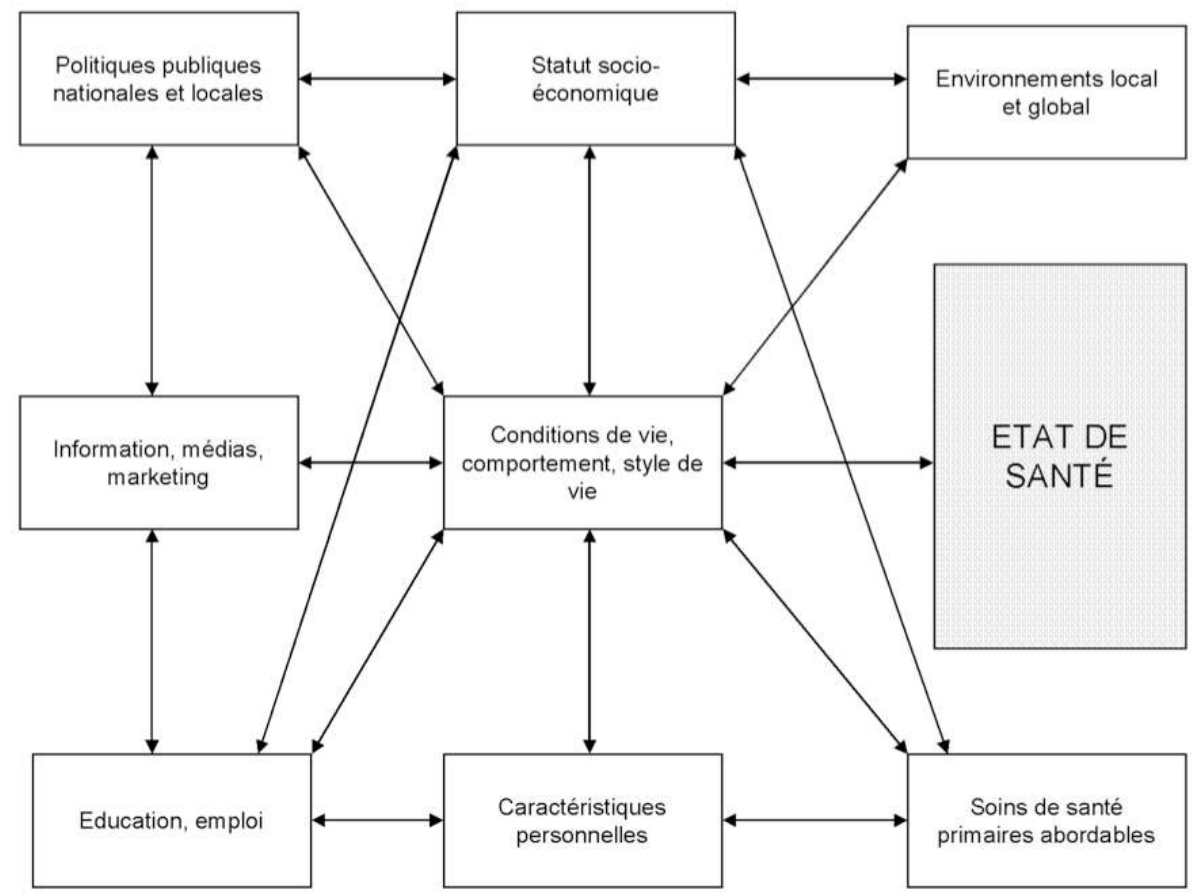

Compte tenu des remarques émises jusqu'à présent, la santé environnementale se caractérise donc par au moins trois principes essentiels. Premièrement, elle se concentre sur la notion de bien-être humain, ce dernier facilitant, tant du point de vue conceptuel que sémantique, une intégration large des déterminants de la santé. Deuxièmement, la santé environnementale repose sur une approche systémique, interdisciplinaire et intersectorielle. En effet, la complexité des interactions entre déterminants de la santé esquissée par exemple par Dahlgren \& Whitehead (1991) implique de faire appel à des disciplines très variées, issues tant du domaine des sciences naturelles que de celui des sciences sociales. En outre, la nature même d'une politique publique de santé environnementale, selon cette approche systémique et interdisciplinaire, requiert un degré élevé d'intersectorialité dans la mesure où l'expertise scientifique nécessaire est généralement dispersée parmi les diverses unités administratives d'un État. Troisièmement, la santé environnementale intègre un nombre variable de déterminants de la santé, fonction du niveau d'inclusion conféré au concept d'environnement.

\section{Conclusion}

Les parcours des concepts de développement durable et de santé environnementale sont donc très divers à plusieurs égards. Sur le plan historique, alors que le développement durable a suivi une complexification croissante à partir de ses prémisses au XVIIIe siècle, la santé environnementale a subi la concurrence intense de la médecine expérimentale, qui, après l'approche intégrée de la santé environnementale défendue par Hippocrate dans l'Antiquité, a concouru, principalement au cours du XIXe siècle, au développement d'une approche sectorielle qui n'a que récemment été remise en cause par les théories 
contemporaines défendant une logique systémique (intégrant notamment des facteurs socioéconomiques) du traitement des problèmes de santé publique.

D'un point de vue éthique, le développement durable a progressivement été récupéré par la sphère politique en tant qu'ensemble de propositions programmatiques dotées d'une forte composante idéologique, rendant toute forme de consensus plus problématique (Hopwood, Mellor and O'Brien, 2005). À l'inverse, la santé environnementale n'a pas nourri, telle quelle, le débat politique. Sa nature apparente, davantage scientifique, circonscrite et consensuelle se prêtant moins à ce genre d'exercice. En revanche, il semble tout à fait pertinent de considérer le développement durable et la santé environnementale en tant que "concepts contestables, disposant de deux niveaux de signification, l'un unitaire mais vague, le second soumis à contestation en raison d'interprétations pratiques divergentes" (Jacobs, 1998). En effet, la santé environnementale n'est pas exempte de conceptualisations très diverses, voire antagonistes, la question de la définition des facteurs environnementaux le démontrant bien. La représentation développée ici plaide évidemment pour un dépassement de conceptions étriquées, faisant principalement de la santé environnementale une chasse gardée des évaluations quantitatives des risques physiques, chimiques ou biologiques.

En conséquence, si les concepts de santé environnementale et de développement durable sont très proches à bien des égards, notamment quant aux principes qui les sous-tendent, ils différent cependant nettement dans l'usage qu'ils supposent en raison d'approches interdisciplinaires antagonistes. Ainsi, la santé environnementale ne saurait être réduite à une composante du développement durable. Sa capacité à inclure un éventail extrêmement large de facteurs environnementaux en lien avec la notion de bien-être humain offre notamment un potentiel considérable en termes de politiques publiques de santé, comprise dans une perspective systémique et intersectorielle vaste.

\section{BIBLIOGRAPHIE}

Ahmed F., 2010, "Tales of adversity", Nature, n468, December, p. 23-30.

Barton H., Mitcham, C., et al., 2003, "Healthy urban planning" in Tsouros A. D. and Farrington J.

L., WHO Healthy Cities in Europe: a compilation of papers on progress and achievements, Copenhagen, WHO/Europe.

Bourdeau P., 2004, "The man-nature relationship and environmental ethics", Journal of Environmental Radioactivity, $\mathrm{n}^{\circ}$ 72, p. 9-15.

Bourdelais P., 2001, Les hygiénistes. Enjeux, modèles et pratiques, Paris, Belin.

Bousson F., 2012, Notre environnement c'est notre santé, Congé-Sur-Orne, Le passager clandestin.

Briggs D., Corvalán C., et al., 1996, Linkage methods for environment and health analysis: General guidelines, Genève, Office of Global and Integrated Environmental Health, World Health Organization.

Carson R. L., 1962, Silent Spring, Cambridge, The Riverside Press. 
Commission mondiale sur l'environnement et le développement, 1987, Notre avenir à tous, Montréal, Éditions du Fleuve.

Dab W., 2007, Santé et environnement, Paris, Presses Universitaires de France.

Dahlgren G. and Whitehead M., 1991, Policies and strategies to promote social equity in health, Stockholm, Institute for Future Studies.

Division for Sustainable Development, 2001, Indicators of Sustainable Development : Framework and Methodologies, New York, Department of Economic and Social Affairs.

Doorslaer E. V., Masseria C., et al., 2006, "Inequalities in access to medical care by income in developed countries", Canadian Medical Association Journal, n 174, p. 2.

Dubos, R., 1966, "Promises and hazards of man's adaptability" in Jarrett H., Environmental quality in a growing economy, Baltimore, Johns Hopkins University Press.

Finnemore M. and Sikkink K., 1998, "International Norm Dynamics and Political Change", International Organization, $\mathrm{n}^{\circ}$ 52, 4, p. 887-917.

Hancock T., 1996, "Planning and Creating Healthy and Sustainable Cities" in Price C. and Tsouros A., Our Cities, Our Future: Policies and Action Plans for Health and Sustainable Development, Copenhagen, WHO Healthy Cities Project Office.

Hopwood B., Mellor M., et al., 2005, "Sustainable Development: Mapping Different Approaches", Sustainable Development, $\mathrm{n}^{\circ} 13$, p. 38-52.

Inglehart R., 1977, The Silent Revolution, Princeton, Princeton University Press.

IUCN, 1980, World Conservation Strategy: Living Resource Conservation for Sustainable Development, IUCN, UNEP, WWF, Gland.

Jacobs M., 1998, "Sustainable Development as a Contested Concept" in Fairness and futurity, Oxford, Oxford University Press, p. 21-45.

Jordan A., 2008, "The governance of sustainable development: taking stock and looking forwards", Environment and Planning C: Government and Policy, n 26, p. 17-33.

Klein J. T., 2004, "Prospects for transdisciplinarity", Futures, n 36, Vol. 4, p. 515-526.

Lalonde M., 1974, A new perspective on the health of Canadians, Ottawa, Minister of National Health and Welfare.

Laughlin S. and Black D., 1995, Poverty and health: tools for change, Birmingham, Public Health Trust.

Lawrence R. J., 2004, "Housing and health: from interdisciplinary principles to transdisciplinary research and practice", Futures, ${ }^{\circ} 36$, p. 487-502.

Ledrans M., 2006, « L'épidémiologie en santé environnementale : Un cadre méthodologique aux applications multiples étroitement lié à celui de l'évaluation des risques sanitaires ", Les Annales des Mines Responsabilité et Environnement, n 41, p. 57-67.

Leeuw E. D., 2009, "Evidence for Healthy Cities: reflections on practice, method and theory", Health Promotion International, $\mathrm{n}^{\circ}$ 24, Vol. S1, p. 19-36.

Lemons, J. and Brown, D. A., 1995. Sustainable development : science, ethics, and public policy, Dordrecht, Kluwer Academic Publishers.

Maslow A. H., 1943, "A Theory of Human Motivation", Psychological Review, n 50, p. 370-396. 
Meadowcroft J., 2007, "National Sustainable Development Strategies: Features, Challenges and Reflexivity", European Environment, n 17, Vol. 3, p. 152-163.

Mebratu D., 1998, "Sustainability and sustainable development : historical and conceptual review", Environmental Impact Assesment Review, n 18, Vol. 6, p. 493-520.

Ministère de la Santé et de la Protection sociale, Ministère de l'Écologie et du Développement durable, et al., 2004, Santé Environnement : Plan national 2004-2008, Paris, Ministère de la Santé et de la Protection sociale.

OFSP and OFEFP, 1997, Plan d'action Environnement et Santé, Berne, OFSP.

OMS, 1946, Constitution de l'Organisation mondiale de la Santé, New York.

OMS, 1978, Les soins de santé primaires : rapport de la Conférence internationale sur les soins de santé primaires, Alma-Ata (URSS), Organisation mondiale de la Santé.

OMS, 1986, Charte d'Ottawa pour la promotion de la santé, Ottawa.

OMS, 1997, Health and Environment in Sustainable Development, Genève, Organisation mondiale de la Santé.

OMS, 1999, Environmental Health Indicators: Framework and Methodologies, Organisation mondiale de la Santé, Genève.

OMS Bureau régional de l'Europe, 1989, Charte européenne de l'environnement et de la santé, Francfort-sur-le-Main, OMS Bureau régional de l'Europe.

OMS Bureau régional de l'Europe, 1990, Environment and Health: The European Charter and Commentary, Copenhagen, WHO/Europe.

OMS Bureau régional de l'Europe, 1991, Les buts de la Santé pour tous : La politique de santé de l'Europe , Copenhague, OMS Bureau régional de l'Europe.

OMS Bureau régional de l'Europe, 1998, Déterminants sociaux de la santé : les faits, Copenhague.

ONU, 1972, Declaration of the United Nations Conference on the Human Environment, Stockholm.

ONU, 1992a, Agenda 21, Rio de Janeiro, Nations-Unies.

ONU, 1992b, Déclaration de Rio sur l'environnement et le développement, Rio de Janeiro, Nations-Unies.

Perdue W. C., Stone L. A., et al., 2003. "The built environment and its relationship to the public's health The Legal Framework", American Journal of Public Health, n 93, Vol. 9.

Repko A. F., 2008, Interdisciplinary Research, Los Angeles, Sage.

Smith, K. R., Corvalan, C. F., et al., 1999, "How much global ill health is attributable to environmental factors?", Epidemiology, n 10 , Vol. 5, p. 573-584.

Tarkowski, S. M., 2007. "Environmental health research in Europe - bibliometric analysis", European Journal of Public Health, $\mathrm{n}^{\circ} 17$.

UN Commission on Sustainable Development, 1996, Indicators of Sustainable Development Framework and Methodologies, New York, UN.

Vos P. D., Malais, G., et al., 2009, "Participation and empowerment in Primary Health Care: from Alma Ata to the era of globalization", Social Medicine, ${ }^{\circ}$ 4, Vol. 2, p. 121-127.

Wikman A., Marklund S., et al., 2005 "Illness, disease, and sickness absence: an empirical test of differences between concepts of ill health", Journal of Epidemial Community Health, $n^{\circ} 59, \mathrm{p}$.

450-454. 
Zaccaï E., 2002, Le développement durable. Dynamique et constitution d'un projet, Bruxelles, P.I.E.-Peter Lang.

Zinsstag J., Schelling E., et al., 2011, "From 'one medicine' to 'one health' and systemic approaches to health and well-being", Preventive Veterinary Medicine, $n^{\circ}$ 101, p. 148-156.

\section{NOTES}

1. Là où le français ne connaît qu'un terme - «maladie » - l'anglais fait la distinction entre "disease", "illness" et "sickness".

2. Voir notamment à ce sujet: Dab, W., 2007. Santé et environnement, Paris, Presses Universitaires de France. (pour un survol historique) ; Lemons J. and Brown D. A., 1995, Sustainable development: science, ethics, and public policy, Dordrecht, Kluwer Academic Publishers. (sur les liens entre développement durable et certains courants philosophiques).

3. Voir par exemple, au sujet de l'hygiénisme: Bourdelais P., 2001, Les hygiénistes. Enjeux, modèles et pratiques, Paris, Belin.

4. "Both aspects of man's environment, the natural and the man-made, are essential to his wellbeing [...]." ONU, 1972. Declaration of the United Nations Conference on the Human Environment, Stockholm.

5. Les dix autres indicateurs concernent principalement la mesure de l'état de santé ("health status"), avec par exemple le taux de mortalité infantile, et des dépenses de santé.

6. Le modèle DPSEEA se concentre principalement sur les pollutions de l'environnement naturel (air, eau, sol, etc.).

7. Les limites de la charge de morbidité environnementale sont importantes, notamment la "difficile prise en compte de certains facteurs de risque environnementaux ayant de longues périodes de latence ou des effets non spécifiques" Prüss-Üstün, A., Mathers, C., et al., 2003. Assessing the environmental burden of disease at national and local levels, Geneva, Organisation Mondiale de la Santé.

8. La question de la complexité de la santé environnementale, qui sera davantage développée dans la suite du texte, repose, d'une part, sur la complexité intrinsèque des concepts de santé et d'environnement, et, d'autre part, sur leur mise en relation.

9. Suivie, en 1998, par l'adoption de la stratégie Santé 21.

10. En 1994, la deuxième Conférence ministérielle sur l'environnement et la santé donne naissance au «Plan d'action en faveur de l'environnement et de la santé dans la région européenne ", suivi par des plans nationaux (NEHAP pour National Environmental Health Action Plan).

11. Il s'agit de notre interprétation schématique de la définition de l'OMS, cette dernière n'en proposant pas.

12. Certains auteurs considèrent cependant le déterminant des soins de santé comme qualitativement très différent des trois autres dans la mesure où il repose davantage sur un concept d'« intervention délibérée ». Leeuw, E. D., 2009, "Evidence for Healthy Cities: reflections on practice, method and theory", Health Promotion International, $\mathrm{n}^{\circ}$ 24, S1, p. 19-36. 


\section{RÉSUMÉS}

Cet article propose une réflexion sur le concept de santé environnementale et sur ses rapports avec celui de développement durable. Alors que les deux concepts ont eu des parcours historiques clairement distincts, nous assistons, depuis la fin des années 1980, à un rapprochement marqué se traduisant la plupart du temps par la tentative d'intégration du concept de santé environnementale dans celui de développement durable. Or, ces deux concepts, s'ils partagent certaines caractéristiques, n'en demeurent pas moins irréductibles l'un à l'autre. De ce fait, en se basant sur les particularités du concept de santé environnementale vis-à-vis de celui de développement durable, cet article vise une reformulation qui permette de lui rendre davantage d'autonomie.

This article proposes a study of the concept of environmental health and of its links with sustainable development. Whereas both concepts have had distinct historical courses, since the end of the 1980's we are witnessing a strong connection resulting mainly in the attempt to integrate the concept of environmental health and that of sustainable development. However, these two concepts, even though they share some common characteristics, still remain basically different. Consequently, this article aims at reformulating the concept of environmental health and its relationship to sustainable development, in order to give it more autonomy.

\section{INDEX}

Mots-clés : santé, environnement, développement durable, interdisciplinarité, institutions

Keywords : health, environment, sustainable development, interdisciplinarity, institutions

\section{AUTEUR}

\section{JULIEN FORBAT}

Julien Forbat, Assistant, thèse de doctorat en cours consacrée aux politiques publiques de santé environnementale en Europe (sous la codirection des professeurs F. Varone, et R. J. Lawrence, Université de Genève), Institut des Sciences de l'Environnement, Université de Genève, julien.forbat@unige.ch 